\title{
Robust Physically-Constrained Modeling of the Mitral Valve and Subvalvular Apparatus
}

\author{
Ingmar Voigt ${ }^{1,2}$, Tommaso Mansi ${ }^{1}$, Razvan Ioan Ionasec ${ }^{1}$, \\ Etienne Assoumou Mengue ${ }^{1}$, Helene Houle ${ }^{3}$, Bogdan Georgescu ${ }^{1}$, \\ Joachim Hornegger ${ }^{2}$, and Dorin Comaniciu ${ }^{1}$ \\ 1 Image Analytics and Informatics, Siemens Corporate Research, Princeton, USA \\ 2 Pattern Recognition Lab, University of Erlangen-Nuremberg, Germany \\ 3 Ultrasound, PLM-Innovation, Siemens Healthcare, Mountain View, CA, USA
}

\begin{abstract}
Mitral valve (MV) is often involved in cardiac diseases, with various pathological patterns that require a systemic view of the entire MV apparatus. Due to its complex shape and dynamics, patient-specific modeling of the MV constitutes a particular challenge. We propose a novel approach for personalized modeling of the dynamic MV and its subvalvular apparatus that ensures temporal consistency over the cardiac sequence and provides realistic deformations. The idea is to detect the anatomical MV components under constraints derived from the biomechanical properties of the leaflets. This is achieved by a robust twostep alternate algorithm that combines discriminative learning and leaflet biomechanics. Extensive evaluation on 200 transesophageal echochardiographic sequences showed an average Hausdorff error of $5.1 \mathrm{~mm}$ at a speed of $9 \mathrm{sec}$, which constitutes an improvement of up to $11.5 \%$ compared to purely data driven approaches. Clinical evaluation on 42 subjects showed, that the proposed fully-automatic approach could provide discriminant biomarkers to detect and quantify remodeling of annulus and leaflets in functional mitral regurgitation.
\end{abstract}

\section{Introduction}

Dysfunctional mitral valves (MV) are found in a large variety of cardiovascular diseases. Mitral valve prolapse for instance, where the leaflets billow into the left atrium due to dysfunctional chordae, is a common cause of regurgitation. A pathological left ventricular (LV) function, like in ischemic or idiopathic dilated cardiomyopathy, can also yield severe mitral valve insufficiency [1] due to an enlarged mitral annulus or abnormal positioning of the papillary muscles. Because the correct functioning of the MV relies on the tight synchronization of the LV, papillaries and leaflets dynamics, its evaluation requires a systemic view of the entire MV apparatus for accurate diagnosis, treatment planning and patient management. Echocardiography is the state-of-the-art modality for MV assessment. However, the complex appearance of the MV, the fast dynamics, the large morphological and functional variations among patients, and the varying image quality (low signal-to-noise ratio, signal dropout, time varying appearance) make the automatic modeling of the MV apparatus very challenging. 
So far, existent valve models are generated manually or, at best, interactively [2. The process is elaborate, tedious, and prone to inter-user variability. A detailed 4D model of the aorta and the MV, but without papillaries, was recently proposed [3]. In this work, the valves are estimated from the images using machine learning and statistical shape models. While these approaches have proven to be robust in many applications [4, their performance may decrease in the presence of image artifacts and in severe pathological morphologies. Furthermore, temporal consistency and physiologically realistic deformations are not guaranteed. In contrast, deformable models integrating prior knowledge about the cardiac deformation have been proposed to estimate the cardiac motion from images 56, with promising results. However, their application for valve dynamics is challenged by the very rapid valve motion. Furthermore, these methods are sensitive to initialization and local minima as their data terms rely on local image features.

Motivated by the above-mentioned limitations, we aim in this paper to estimate a personalized model of the mitral valve that is temporally and physiologically consistent. To that end, we propose a novel discriminative learning technique that is constrained by a biomechanical model of the MV leaflets. The model is automatically initialized in the images and local minima are avoided through incremental search and discriminative learning. The biomechanical constraint is ensured by solving a dynamic system between time frames. Besides, our model includes the MV annulus, anterior and posterior leaflets, and the subvalvular apparatus represented by landmarks at the papillary tips. To the best of our knowledge this is the first time that machine learning methods are integrated with biomechanical constraints. Extensive evaluation on 200 transesophageal echochardiographic (TEE) sequences show an average Hausdorff error of $5.1 \mathrm{~mm}$ at a speed of $9 \mathrm{sec}$ per volume, which constitutes an improvement of up to $11.5 \%$ compared to state-of-the-art approaches [37], which are purely data driven. A clinical evaluation on 42 subjects is carried out, showing that our method can provide reliable discriminant biomarkers to detect and quantify remodeling of MV shape and dynamics.

\section{Methods}

\subsection{General Overview of the Algorithm}

Our goal is to estimate a physiologically realistic 4D model of the MV and its subvalvular apparatus from $3 \mathrm{D}+\mathrm{t}$ cardiac images. The idea is to constrain a machine learning approach such that the estimated point distribution model $S \in$ $\Omega, \Omega$ being the space of physiological surface shapes, satisfies MV biomechanics. Although the proposed approach is applied on the entire $4 \mathrm{D}$ time series, in the following we describe the algorithm between two frames $I_{t_{1}}$ and $I_{t_{2}}$, at time $t_{1}$ and $t_{2}$ only, for the sake of clarity. The constrained problem is written as: 

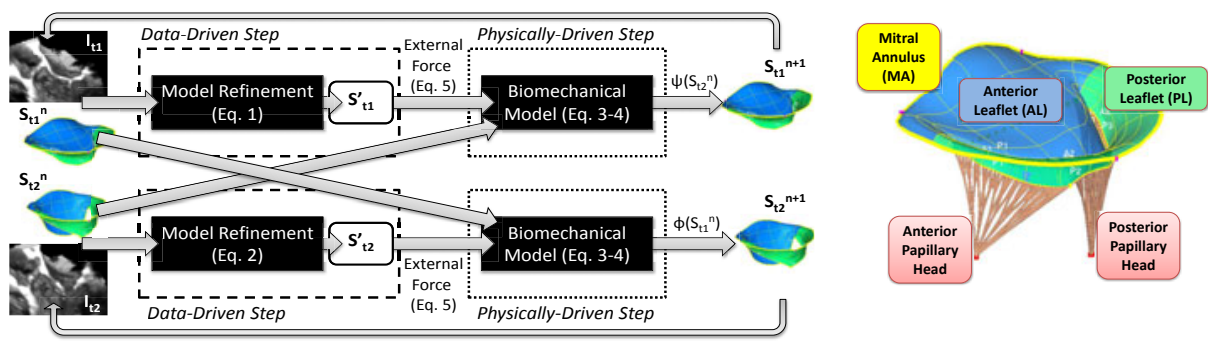

Fig. 1. Left panel: Proposed framework for physically-constrained MV estimation. See text for details. Right panel: Mitral valve apparatus model in systole.

$$
\left\{\begin{array}{l}
S_{t_{1}}=\arg \underset{\hat{S} \in \Omega}{\max } p\left(\hat{S} \mid I_{t_{1}}\right) \\
S_{t_{2}}=\arg \underset{\hat{S} \in \Omega}{\max } p\left(\hat{S} \mid I_{t_{2}}\right) \\
S_{t_{2}}=\phi\left(S_{t_{1}}\right) \text { and } S_{t_{1}}=\psi\left(S_{t_{2}}\right)
\end{array}\right.
$$

where $p$ is a discriminative probabilistic model and $\phi$ and $\psi$ are the physiological deformations calculated by solving a dynamic system. To achieve computational efficiency, we solve this high-dimensional constrained and coupled problem with a two-step optimization procedure, as illustrated in Fig. 1 At a given iteration $n$ of the algorithm loop, let $S_{t_{1}}^{n}$ and $S_{t_{2}}^{n}$ be the current physically constrained models in $I_{t_{1}}$ and $I_{t_{2}}$ respectively. In the first "data-driven" step, we refine $S_{t_{1}}^{n}$ using discriminative machine learning on $I_{t_{1}}$ to obtained a new estimate $S_{t_{1}}^{\prime}$ (Eq. 1). In the second "physically-driven" step, we deform $S_{t_{2}}^{n}$ according to a biomechanical model $\psi$ with external force calculated from $S_{t_{1}}^{\prime}$ to obtain an updated physicallyconstrained $S_{t_{1}}^{n+1}$ (Eq. 3) Thus, the updated model in image $I_{t_{1}}$ is a deformed realization of $S_{t_{2}}^{n}$ from image $I_{t_{2}}$ subject to data-driven information related to $I_{t_{1}} \cdot S_{t_{2}}^{n+1}$ is computed in parallel following the same procedure. These two-steps, further detailed below, are iterated until convergence.

\subsection{Data-Driven Step: Estimation of Mitral Valve Apparatus Model}

Anatomical Model Parameters. The anatomical model $S$ of the MV and its subvalvular component comprises: the mitral annulus (MA), the anterior and posterior leaflets and the anterior and posterior papillary heads (Fig. 1 right). More precisely, $S$ is a point distribution model consisting of 986 points and 1792 triangles with consistent parameterization derived from anatomical landmarks (three trigones, three commissures, two leaflet tips and two papillary heads) 3 .

Learning-Based Spatial Estimation. The parameters of the anatomical model $S$ are estimated from the images using a hierarchical discriminative learning algorithm as in 3] (Eq. 1] and Eq. 2). The conditional probability $p(S \mid I)$ is incrementally modeled within the Marginal Space Learning (MSL) framework, 
which consists in training and detecting on marginal spaces using the Probabilistic Boosting Tree (PBT) with Haar and steerable features [7]. Different detectors are employed for the mitral annulus and free-edges contours and the leaflet surfaces to improve detection accuracy.

\subsection{Physically-Driven Step: Biomechanical Constraint}

We now constrain the estimations in $I_{t_{1}}$ and $I_{t_{2}}$ according to a biomechanical model of the MV leaflets. Let $S_{t_{1}}^{n}$ and $S_{t_{2}}^{n}$ be the current physically-constrained model and $S_{t_{1}}^{\prime}$ and $S_{t_{2}}^{\prime}$ the new estimates computed as described in the previous sections. The updated constrained model $S_{t_{1}}^{n+1}$ related to $I_{t_{1}}$ is obtained by deforming $S_{t_{2}}^{n}$ towards $S_{t_{1}}^{\prime}$. This deformation, $\psi$ in Eq. 3, is achieved by solving the dynamic system

$$
\mathrm{M} \ddot{\mathbf{U}}+\mathrm{C} \dot{\mathbf{U}}+\mathrm{K} \mathbf{U}=\mathbf{F}_{e x t}
$$

$\mathbf{U}$ is the displacement vector of the vertices of $S_{t_{2}}^{n}$, $\mathbf{U}$ their velocity and $\ddot{\mathbf{U}}$ their acceleration. $\mathrm{M}$ is a diagonal mass matrix (leaflet mass density $\rho=1.04 \mathrm{~g} / \mathrm{mL}$ ), $\mathrm{C}$ is a Rayleigh damping matrix, $\mathrm{C}=0.1(\mathrm{M}+\mathrm{K})$, and $\mathrm{K}$ is the stiffness matrix of the internal elastic forces. We approximate the tissue properties of the leaflets by a linear isotropic material to optimize computational efficiency for fast estimation. The leaflet thickness is set to $2 \mathrm{~mm}$, the average thickness observed in our datasets. Near-incompressibility is achieved with a Poisson ratio $\nu$ of 0.488 and a Young modulus $E$ of $6.2 M P a$ is used [8].

$\mathbf{F}_{\text {ext }}$ is the external force that drives $S_{t_{2}}^{n}$ towards the new estimate $S_{t_{1}}^{\prime}$. We want the result to be as close as possible to $S_{t_{1}}^{\prime}$ but that preserves the tangential motion generated by the internal forces. We therefore move the vertices $\mathbf{v}_{t_{2}}^{n}$ of $S_{t_{2}}^{n}$ along their normal direction $\mathbf{n}$, towards their corresponding vertex $\mathbf{v}_{t_{1}}^{\prime}$ in $S_{t_{1}}^{\prime}$. Furthermore, we weight that force according to the uncertainty in the data term $p\left(\mathbf{v}_{t_{1}}^{\prime} \mid I_{t_{1}}\right)$ such that positions with low confidences have little influence on the leaflet deformation, while high confidences result in high influence. Thus, $\mathbf{F}_{\text {ext }}$ is written as:

$$
\mathbf{F}_{e x t}\left(\mathbf{v}_{t_{2}}^{n}\right)=-\kappa p\left(\mathbf{v}_{t_{1}}^{\prime} \mid I_{t_{1}}\right)\left(\mathbf{v}_{t_{1}}^{\prime}-\mathbf{v}_{t_{2}}^{n}\right) \cdot \mathbf{n}
$$

$\kappa$ is a weight parameter, which in our experiments we empirically set to 0.1 . The vertices $\mathbf{v}_{t_{2}}^{n}$, and thus the force $\mathbf{F}_{\text {ext }}$, are updated at every time step of the resolution of the dynamic system in Eq. 4. which is solved using co-rotational triangular finite element methods (FEM) to cope with large deformations and rotations [9]. An implicit Euler solver is employed to update mesh positions. The deformation ends when the average relative displacement of the surface vertices is lower than the image resolution (typically $1 \mathrm{~mm}$ ).

Intuitively, the final deformed model $\psi\left(S_{t_{2}}^{n}\right)=S_{t_{1}}^{n+1}$ matches the estimated model $S_{t_{1}}^{\prime}$. However, here, the new model $S_{t_{1}}^{n+1}$ takes into account the internal forces and is robust to outliers with low confidence from the data term. It is also physically consistent with $S_{t_{2}}^{n}$. We apply the same procedure, symmetrically, to estimate $S_{t_{2}}^{n+1}$, and iterate. The algorithm is extended to $4 \mathrm{D}$ sequences by propagating the models over the cardiac sequence using a cyclic constraint and 
Table 1. Estimation accuracy in terms of maximum (Hausdorff) and average (pointto-mesh) distances in $\mathrm{mm}$ to the ground truth for the biomechanically constrained and unconstrained, purely data driven approaches. Notable improvements are highlighted in bold faced font for corresponding metrics for both constrained and unconstrained estimation results.

\begin{tabular}{|c|c|c|c|c|c|c|c|c|c|c|c|c|}
\hline & \multicolumn{6}{|c|}{ Max error (Hausdorff) } & \multicolumn{6}{|c|}{ Average error (Point-to-mesh) } \\
\hline & & istr & & & 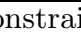 & & cons & & & uncor & & ind \\
\hline & $n$ & std & $0 \%$ & me & $\mathrm{td}$ & & $\mathrm{m}$ & & $\overline{n g}$ & & & \\
\hline & & & & & 1.79 & & & & 2.54 & & & \\
\hline e & 32 & 1.85 & 7.53 & 5.73 & 2.69 & 8. & 2.44 & 0.70 & 3.32 & 39 & 0.85 & 3.2 \\
\hline $\mathrm{Le}$ & 5.57 & 1.68 & 7.51 & 5.70 & 2.49 & 8.36 & 3.11 & 1.02 & 4.14 & 3.02 & 1.11 & 4.3 \\
\hline
\end{tabular}

iterating until convergence. The algorithm ends when the norm of the maximum update displacement in all the frames is below the image resolution. In practice, the proposed algorithm converged fairly fast. This may be explained by the fact that each surface is deformed using the other estimated model. As the model comes closer to the maximum PBT, an equilibrium is found.

\section{$3 \quad$ Experimental Results}

The evaluation of the proposed method splits into two subparts: $i$ ) the quantitative evaluation of the model estimation performance and ii) a clinical evaluation in terms of a predictive biomarker for diagnosis and therapy planning.

Estimation performance. Our method has been evaluated using a comprehensive set of 200 4D TEE studies from 120 patients, which were acquired using different capture ranges and image resolutions. Each study was associated with ground-truth models, which were manually fitted to the image data. The performance results were obtained using three-fold cross validation experiments for both biomechanically constrained and unconstrained estimation schemes. The method performs with a speed of 9 seconds per volume on a standard desktop machine (Intel Core Duo 2.66 GHz, 2 GB RAM). The biomechanical deformations required 5 to 25 steps to converge. The overall algorithm converged in 5 to 7 iterations and no convergence problems could be observed. The average point-to-mesh distance and Hausdorff distance are reported in Table 1.

While the average point-to-mesh accuracy is comparable for both methods considering the $1 \mathrm{~mm}$ image resolution -, the Haussdorf distances underlines the specific strength of the biomechanical constraint by providing improved robustness over the purely data driven approach esp. in cases with challenging image quality and constitutes an improvement of up to $11.5 \%$, while at the same time preserving a reasonable estimation speed of 9 seconds per volume. Particularly the leaflets and free edges of the valve, which are governed by rapid motion and signal dropout - esp. in diastole - benefit from the constrained estimation scheme as indicated by a reduced mean, standard deviation and 90-percentile. Fig. 2 illustrates a specific case for which the unconstrained estimation algorithm fails 


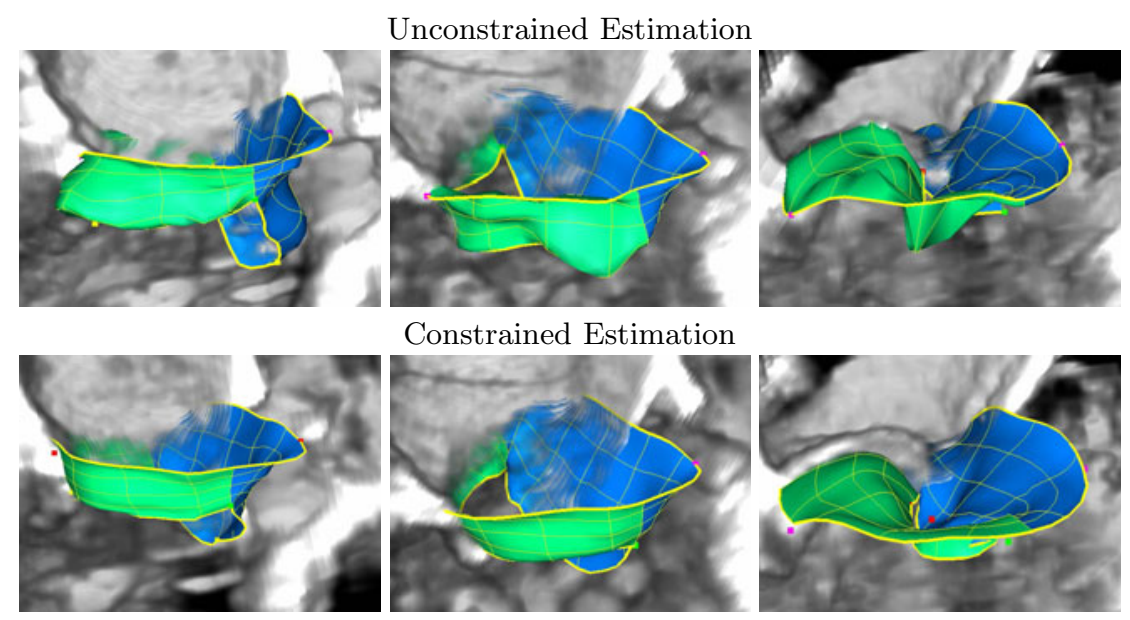

Fig. 2. Exemplary estimation results on three patients. While the unconstrained estimation alone is subject to image noise and signal dropout, the biomechanical constraint is compensating for such outliers and provides realistic shapes and smooth surfaces.

to delineate the mitral valve due to image noise, while the biomechanical constraint compensates for such outliers and provides realistic shapes and smooth surfaces. As the proposed algorithm utilizes a biomechanical model of the valves, temporal consistency and physiological variation in anatomical dimensions respects the near-incompressibility of the tissue. The relative temporal variation in surface area and leaflet length is consistently lower using the biomechanical constraint and quantifies to $10 \%$ vs. $14 \%$ for the constrained vs. unconstrained estimation.

Clinical Evaluation. The contributions of mitral annular (MA) and leaflet (ML) remodeling to functional mitral regurgitation (FMR) is a key factor in the consideration of mitral valve annuloplasty. Selection of the synthetic ring size and type is based on the quantification of MA and ML remodeling. We thus tested the feasibility of the proposed MV modeling approach to quantify MA and ML remodeling in FMR [10.

The mitral valve was estimated from 15 healthy subjects and 27 patients with moderate or severe FMR. Among the patients, 12 presented with normal annulus size (Group 1) and 15 with a dilated annulus (Group 2). From the 4D anatomical model of the MV, we calculated different metrics reported in Table 2. The dynamic change of the antero-posterior (AP) annular diameter, computed as the difference diameter between early systole and early diastole, significantly discriminated the normals from FMR Group 1 (Fig. 3). In particular, it indicates a reduced accentuation of the saddle shape with consequent reduction in leaflet coaptation. In addition to a dilated, akinetic annulus, the anterior surface length was markedly increased in FMR Group 2, which charaterizes significant ML remodeling. The choice of ring size and type can be made based on these 
Table 2. Model-based measurements for quantification of functional mitral regurgitation (FMR) and surgical planning: Mean and standard deviation in respective groups reported in $\mathrm{mm}$.

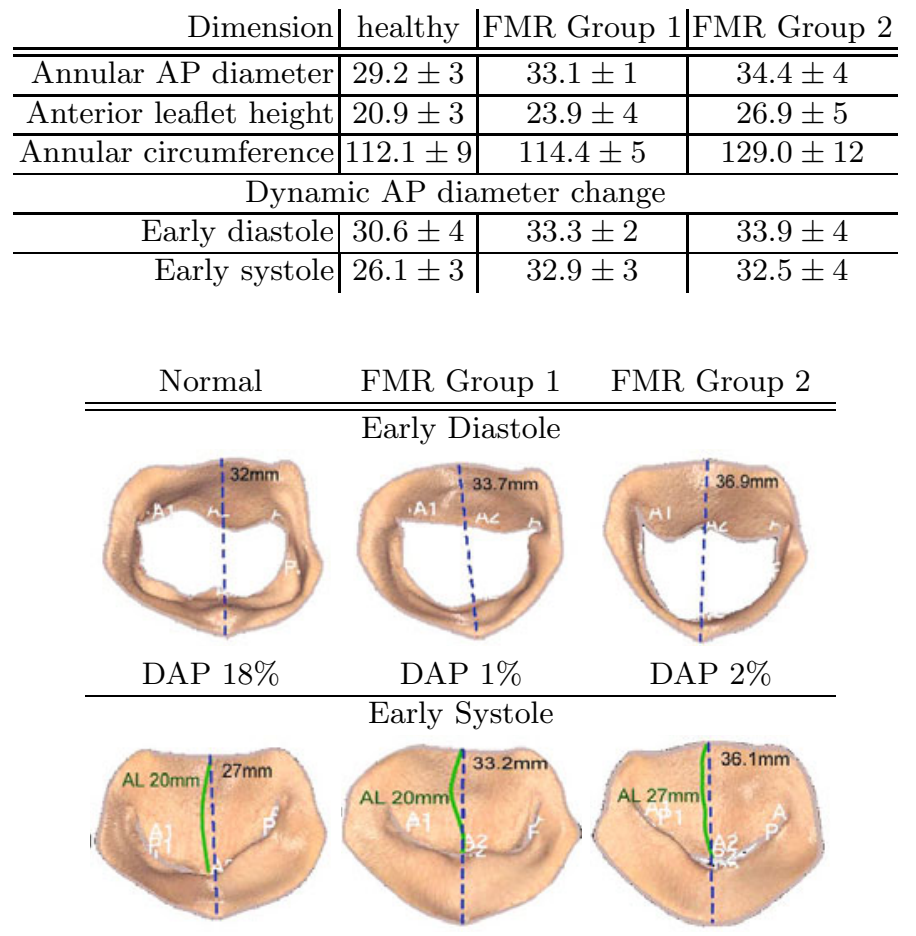

Fig. 3. Dynamic AP (DAP) Diameter (blue dashed line) varies by $18 \%$ over the cardiac cycle in healthy subjects in contrast to FMR cases (DAP $=1-2 \%$ ). Anterior leaflet height (green solid line) was normal in group 1 but not in group 2, indicating predominant annular remodeling in that population [10. Reproduced with permission of the authors.

automated measurements. We can thus conclude that automated 3-D quantitative surgical anatomy in FMR 1) shows that an akinetic annulus is an early basis for MR, followed by annular dilatation and anterior leaflet lengthening, and 2) that these mechanistic insights and the quantitative characterization of the pathological anatomy can aid surgical decision-making.

\section{Conclusion}

We proposed a novel approach for physiologically and temporally compliant estimation of the mitral valve by combining discriminative learning methods with biomechanical constraints. The data-driven step ensures efficiency and robustness while the physically-driven step guarantees physiological consistency. Our 
method is fully automatic, robust to initialization and image artifacts. Furthermore, our mitral valve model comprises the mitral annulus, anterior and posterior leaflets and for the first time, the subvalvular apparatus. Quantitative evaluation on 200 datasets demonstrated that our constrained method outperforms purely data driven approaches by $11.5 \%$ in terms of model estimation (average Hausdorff error of $5.1 \mathrm{~mm}$ ). Clinical evaluation demonstrated that the proposed approach could provide reliable biomarkers for mitral annulus and leaflets remodeling in functional mitral regurgitations. Future work will further investigate effects of the different degrees of freedom such as anistropic and non-linear material properties.

\section{References}

1. Borger, M., Alam, A., Murphy, P., Doenst, T., David, T.: Chronic ischemic mitral regurgitation: Repair, replace or rethink? Ann. Thorac. Surg. (81), 1153-1161 (2006)

2. Votta, E., Caiani, E., Veronesi, F., Soncini, M., Montevecchi, F., Redaelli, A.: Mitral valve finite-element modelling from ultrasound data: a pilot study for a new approach to understand mitral function and clinical scenarios. Philosophical Transactions of the Royal Society A: Mathematical, Physical and Engineering Sciences 366(1879), 3411 (2008)

3. Ionasec, R., Voigt, I., Georgescu, B., Wang, Y., Houle, H., Vega-Higuera, F., Navab, N., Comaniciu, D.: Patient-Specific Modeling and Quantification of the Aortic and Mitral Valves From 4-D Cardiac CT and TEE. IEEE Transactions on Medical Imaging 29(9), 1636-1651 (2010)

4. Heimann, T., Meinzer, H.P.: Statistical shape models for 3d medical image segmentation: A review. Medical Image Analysis 13(4), 543-563 (2009)

5. Papademetris, X., Sinusas, A.J., Dione, D.P., Duncan, J.S.: Estimation of 3D left ventricular deformation from echocardiography. Med. Image Anal. 5, 17-28 (2001)

6. Sermesant, M., Delingette, H., Ayache, N.: An electromechanical model of the heart for image analysis and simulation. IEEE Transactions in Medical Imaging 5(25), 612-625 (2006)

7. Zheng, Y., Barbu, A., Georgescu, B., Scheuering, M., Comaniciu, D.: Four-chamber heart modeling and automatic segmentation for 3 -d cardiac ct volumes using marginal space learning and steerable features. IEEE Transactions on Medical Imaging 27(11), 1668-1681 (2008)

8. Schievano, S., Kunzelman, K., Nicosia, M., Cochran, R., Einstein, D., Khambadkone, S., Bonhoeffer, P.: Percutaneous mitral valve dilatation: Single balloon versus double balloon. a finite element study. Journal of Heart Valve Disease 18(1), 28-34 (2009)

9. Nesme, M., Payan, Y., Faure, F.: Efficient, physically plausible finite elements. Eurographics (short papers), 77-80 (2005)

10. Calleja, A., Stiver, K., Thavendiranathan, P., Liu, S., Ionasec, R., Voigt, I., Houle, H., Michelis, N.D., Ryan, T., Vannan, M.: Automated Quantitative 3-D Echocardiography of The Surgical Mitral Valve Anatomy in Functional Mitral Regurgitation to Guide Mitral Valve Repair. In: Proceedings of the 22nd Annual Scientific Sessions of the American Society of Echocardiography (2011) 